\title{
Comparison of MacNeille, Canonical, and Profinite Completions
}

\author{
Guram Bezhanishvili • Jacob Vosmaer
}

Received: 5 January 2008 / Accepted: 3 September 2008 / Published online: 1 October 2008

(C) The Author(s) 2008. This article is published with open access at Springerlink.com

\begin{abstract}
Using duality theory, we give necessary and sufficient conditions for the MacNeille, canonical, and profinite completions of distributive lattices, Heyting algebras, and Boolean algebras to be isomorphic.
\end{abstract}

Keywords MacNeille completion - Canonical completion • Profinite completion • Duality theory

Mathematics Subject Classifications (2000) $06 \mathrm{~B} 23 \cdot 06 \mathrm{D} 20 \cdot 06 \mathrm{D} 50$

\section{Introduction}

In the theory of lattice completions and in applications of lattice theory to logic, the MacNeille and canonical completions play a fundamental role. The MacNeille completions provide completeness of various predicate logics with respect to their algebraic semantics, as was shown in $[16,17,19]$. On the other hand, the JónssonTarski representation theorem for Boolean algebras with operators [14] is the key for many completeness results in non-classical propositional logics (such as modal and superintuitionistic logics). A connection between the MacNeille and

The second author was supported by VICI grant 639.073.501 of the Netherlands Organization for Scientific Research (NWO).

G. Bezhanishvili

Department of Mathematical Sciences,

New Mexico State University, Las Cruces, NM 88003-8001, USA

e-mail: gbezhani@nmsu.edu

J. Vosmaer $(\bowtie)$

Institute for Logic, Language and Computation,

University of Amsterdam, Plantage Muidergracht 24,

1018 TV Amsterdam, The Netherlands

e-mail: J.Vosmaer@uva.nl 
canonical completions was discussed in [9]. Yet another completion-the profinite completion-has its origins in Galois theory. It has recently been shown that there is a close connection between the canonical and profinite completions [3, 12]. The aim of this paper is to compare these three completions for distributive lattices, Heyting algebras, and Boolean algebras. Our main tool is duality theory.

We give a necessary and sufficient condition for the MacNeille completion $\bar{L}$ of a bounded distributive lattice $L$ to be isomorphic to its canonical completion $L^{\sigma}$. Since $L^{\sigma}$ is isomorphic to the profinite completion $\widehat{L}$ of $L$ [3, Theorem 2.11], we obtain a criterion for the three completions to be isomorphic. The isomorphisms, however, may not commute with the embeddings $\eta: L \hookrightarrow \bar{L}, \zeta: L \hookrightarrow L^{\sigma}$, and $\iota: L \hookrightarrow \widehat{L}$. We give necessary and sufficient conditions for the isomorphisms to commute with $\eta, \zeta$, and $\iota$. When $L$ happens to be a Heyting algebra, our results about the isomorphism between $\bar{L}$ and $L^{\sigma}$ apply unchanged. But now, unlike the case of bounded distributive lattices, $L^{\sigma}$ is not necessarily isomorphic to $\widehat{L}$. Therefore, we add necessary and sufficient conditions for the existence of an isomorphism between $L^{\sigma}$ and $\widehat{L}$, and between $\bar{L}$ and $\widehat{L}$. Consequently, we obtain necessary and sufficient conditions for the three completions of a Heyting algebra to be isomorphic. We also give necessary and sufficient conditions for these isomorphisms to commute with $\eta, \zeta$, and $\iota$.

The paper is organized as follows. In Section 2 we recall the definitions and basic facts about the MacNeille, canonical, and profinite completions. In Section 3 we recall the Priestley duality for bounded distributive lattices, and then establish our first main results about the isomorphism of the MacNeille, canonical, and profinite completions of bounded distributive lattices. In Section 4 we briefly recall the Esakia duality for Heyting algebras, and then prove our main results about the isomorphism of the three completions for Heyting algebras. We also discuss consequences of our results for the case of Boolean algebras. Finally, in Section 5 we briefly discuss extensions of our results to more general settings.

\section{Preliminaries}

In this paper we are interested in the conditions under which different completions of distributive lattices and related algebras are isomorphic. We are mostly concerned with the following three completions: the MacNeille completion, the canonical completion, and the profinite completion. In this preliminary section we introduce the main definitions from the theory of completions of lattices to make the paper self-contained.

Let $P$ be a poset. For $a \in P$ let $\uparrow a=\{x \in P \mid a \leq x\}$ and $\downarrow a=\{x \in P \mid x \leq a\}$. We recall that $S \subseteq P$ is join-dense in $P$ if for all $a \in P$ we have $\bigvee(\downarrow a \cap S)$ exists and $a=\bigvee(\downarrow a \cap S)$. The notion of a meet-dense subset of $P$ is dual. The next lemma is well-known (see, e.g., [6, p. 160]).

Lemma 2.1 Let L be a lattice and let $S \subseteq L$.

(1) $S$ is join-dense in $L$ iff for each $a, b \in L$ with $a \not \leq b$, there exists $s \in S$ such that $s \leq a$ and $s \not \leq b$.

(2) $S$ is meet-dense in L iff for each $a, b \in L$ with $a \not \leq b$, there exists $s \in S$ such that $a \not \leq s$ and $b \leq s$. 
Throughout we assume that all lattices are bounded. If not, then we can always add a new top and bottom to them. The next definition is well-known; see, e.g., [15, 21]. Note that we do not assume that $L$ is a complete lattice.

Definition 2.2 Let $L$ be a lattice.

(1) For $p, q \in L$ we say that a pair $(p, q)$ splits $L$ if $\uparrow p \cap \downarrow q=\emptyset$ and $\uparrow p \cup \downarrow q=L$. When $(p, q)$ splits $L$, then we call $(p, q)$ a splitting pair, $p$ a splitting element, and $q$ a co-splitting element. Let $S(L)$ denote the set of splitting elements of $L$ and $C S(L)$ denote the set of co-splitting elements of $L$.

(2) We say that $0 \neq p \in L$ is join-prime if from $p \leq a \vee b$ it follows that $p \leq a$ or $p \leq b$. The notion of a meet-prime element is dual. Let $J(L)$ and $M(L)$ denote the sets of join-prime and meet-prime elements of $L$, respectively.

(3) We say that $0 \neq p \in L$ is completely join-prime if for each $S \subseteq L$ such that $\bigvee S$ exists and $p \leq \bigvee S$, there exists $s \in S$ with $p \leq s$. The notion of a completely meet-prime element is dual. Let $J^{\infty}(L)$ and $M^{\infty}(L)$ denote the sets of completely join-prime and completely meet-prime elements of $L$, respectively.

It is easy to see that $S(L) \subseteq J^{\infty}(L) \subseteq J(L)$ and that $C S(L) \subseteq M^{\infty}(L) \subseteq M(L)$. Moreover, if $L$ is complete, then $S(L)=J^{\infty}(L)$ and $C S(L)=M^{\infty}(L)$. To see this, observe that from $p \in J^{\infty}(L)$ (resp. $\left.q \in M^{\infty}(L)\right)$ it follows that $(p, \bigvee\{x \in L \mid p \not \leq x\})$ (resp. $(\bigwedge\{x \in L \mid x \not \leq q\}, q)$ ) is a splitting pair, and so $p \in S(L)$ (resp. $q \in C S(L))$. Furthermore, if $L$ is finite, then it is obvious that in addition we have $J^{\infty}(L)=J(L)$ and $M^{\infty}(L)=M(L)$. An example of a complete lattice in which $J^{\infty}(L) \subset J(L)$ (resp. $M^{\infty}(L) \subset M(L)$ ) is the lattice of closed (resp. open) subsets of the real line. Indeed, singletons (resp. complements of singletons) are join-prime (resp. meet-prime), but are not completely join-prime (resp. completely meet-prime). An example of an incomplete lattice in which $S(L) \subset J^{\infty}(L)\left(\operatorname{resp} . C S(L) \subset M^{\infty}(L)\right)$ is given below.

Example 2.3 Let $\omega$ be the first infinite ordinal with its usual order, and consider the (distributive) lattice $L=(\omega \times \omega) \oplus \omega^{\mathrm{op}}$, where $\oplus$ is the operation of sum and $\omega^{\mathrm{op}}$ is the opposite of $\omega$ (see Fig. 1). Clearly $L$ is not complete. Moreover, each $p=(0, n)$ or $(n, 0)$ is in $J^{\infty}(L)$, but does not belong to $S(L)$. By taking the opposite of $L$ we obtain a lattice in which not every completely meet-prime element is co-splitting.

Definition 2.4 Let $L$ be a lattice.

(1) $[5$, p. 211] We call $L$ principally separated if for each $a, b \in L$ with $a \not \leq b$, there is a splitting pair $(p, q)$ such that $p \leq a$ and $b \leq q$.

(2) We call L completely join-prime generated if $J^{\infty}(L)$ is join-dense in $L$.

Remark 2.5 Using Lemma 2.1, it is easy to see that if $L$ is principally separated, then $S(L)$ is join-dense in $L$. Since $S(L) \subseteq J^{\infty}(L)$, it follows that each principally separated lattice is completely join-prime generated. On the other hand, the lattice given in Example 2.3 is an example of a completely join-prime generated lattice, which is not principally separated. Nevertheless, the observations in the paragraph following Definition 2.2 imply that the two notions are equivalent in the setting of complete lattices. 
Fig. 1 Example 2.3
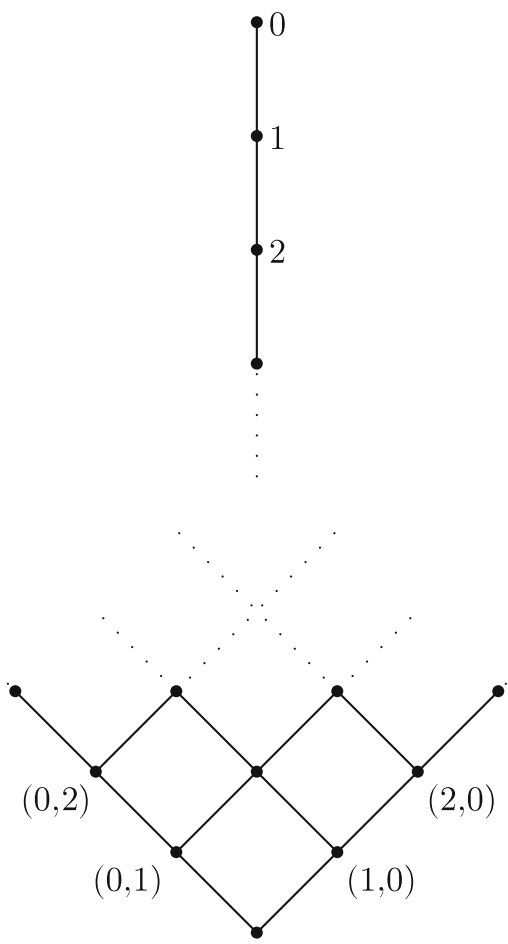

$(0,0)$

Definition 2.6 Let $L$ be a lattice. A completion of $L$ is a pair $(C, \xi)$ where $C$ is a complete lattice and $\xi: L \rightarrow C$ is a lattice embedding. Given two completions of $L,(C, \xi)$ and $(D, \mu)$, and a lattice isomorphism $f: C \rightarrow D$, we call $f:(C, \xi) \rightarrow$ $(D, \mu)$ an isomorphism of completions if $f \circ \xi=\mu$. We say $(C, \xi)$ and $(D, \mu)$ are isomorphic as completions of $L$ if there exists an isomorphism of completions $f:(C, \xi) \rightarrow(D, \mu)$.

Now we recall the definitions of the MacNeille and canonical completions of lattices.

Definition 2.7 Let $L$ be a lattice.

(1) [1] The MacNeille completion of $L$ is a pair $(\bar{L}, \eta)$, where $\bar{L}$ is a complete lattice and $\eta: L \rightarrow \bar{L}$ is a lattice embedding such that $\eta[L]$ is both join-dense and meet-dense in $\bar{L}$.

(2) [8] The canonical completion of $L$ is a pair $\left(L^{\sigma}, \zeta\right)$, where $L^{\sigma}$ is a complete lattice and $\zeta: L \rightarrow L^{\sigma}$ is a lattice embedding such that:

(i) For each filter $F$ and ideal $I$ of $L$, from $F \cap I=\emptyset$ it follows that $\wedge \zeta[F] \not \leq$ $\bigvee \zeta[I]$. 
(ii) The set $K_{L}=\{\bigwedge \zeta[S] \mid S \subseteq L\}$ of closed elements is join-dense in $L^{\sigma}$.

(iii) The set $O_{L}=\{\bigvee \zeta[S] \mid S \subseteq L\}$ of open elements is meet-dense in $L^{\sigma}$.

The third completion we will be dealing with in this paper is the profinite completion. Let $L$ be a lattice and let $\Phi_{L}=\{\theta \in \operatorname{Con} L \mid L / \theta$ is finite $\}$ be the congruences of $L$ of finite index. For $\theta, \psi \in \Phi_{L}$ with $\theta \subseteq \psi$, let $h_{\theta \psi}: L / \theta \rightarrow L / \psi$ be given by $h_{\theta \psi}(a / \theta)=a / \psi$. Then $\left(\Phi_{L}, \supseteq\right)$ is a directed poset, and so $\left\langle\{L / \theta\}, h_{\theta \psi}\right\rangle$ is an inverse system of finite homomorphic images of $L$. We denote the inverse limit of $\left\langle\{L / \theta\}, h_{\theta \psi}\right\rangle$ by $\widehat{L}$. It is well-known that

$$
\widehat{L}=\left\{\alpha \in \prod_{\Phi_{L}} L / \theta \mid \forall \theta, \psi \in \Phi_{L} \text { with } \theta \subseteq \psi \text {, if } \alpha(\theta)=a / \theta \text { then } \alpha(\psi)=a / \psi\right\} .
$$

We define $\iota: L \rightarrow \widehat{L}$ by $\iota(a)=\alpha_{a}$, where $\alpha_{a}(\theta)=a / \theta$ for $\theta \in \Phi_{L}$. We note that $\iota: L \rightarrow \widehat{L}$ may not be $1-1$ in general, but $\iota$ is $1-1$ if $L$ is distributive (see, e.g., [3, pp. 145-146]).

Definition 2.8 [3] Let $L$ be a distributive lattice. The profinite completion of $L$ is the pair $(\widehat{L}, \iota)$.

In this paper we are primarily concerned with two questions. Firstly, we want to find necessary and sufficient conditions for the lattices $\bar{L}, L^{\sigma}$, and $\widehat{L}$ to be isomorphic. Secondly, we seek necessary and sufficient conditions for the completions $(\bar{L}, \eta)$, $\left(L^{\sigma}, \zeta\right)$, and $(\widehat{L}, \iota)$ to be isomorphic as completions of $L$.

\section{Distributive Lattices}

We briefly recall the basics of Priestley duality [18] for distributive lattices. For a topological space $X$, let $\mathbf{I}$ and $\mathbf{C}$ denote the interior and closure operators of $X$, respectively. We recall that a subset $U$ of $X$ is clopen if $U$ is both closed and open, and that $X$ is zero-dimensional if clopen subsets of $X$ form a basis for the topology. We also recall that $X$ is a Stone space if $X$ is compact, Hausdorff, and zero-dimensional.

Let $\langle X, \leq\rangle$ be a partially ordered set. We call $A \subseteq X$ an upset of $X$ if $x \in A$ and $x \leq y$ imply $y \in A$. Let $\operatorname{Up}(X)$ denote the set of upsets of $X$.

Definition 3.1 A Priestley space is a triple $\langle X, \tau, \leq\rangle$, where $\langle X, \tau\rangle$ is a compact space, $\langle X, \leq\rangle$ is a partially ordered set, and $\langle X, \tau, \leq\rangle$ satisfies the Priestley separation axiom: For $x, y \in X$ with $x \not \leq y$, there exists a clopen upset $U$ of $X$ such that $x \in U$ and $y \notin U$. (Note that the above conditions imply that $\langle X, \tau\rangle$ is a Stone space.)

By Priestley duality, the dual of a distributive lattice $L$ is the Priestley space $\left\langle\operatorname{Pr}(L), \tau_{L}, \subseteq\right\rangle$, where $\operatorname{Pr}(L)$ is the set of prime filters of $L$ and $\tau_{L}$ is the topology generated by $\{\varphi(a)-\varphi(b) \mid a, b \in L\}$; here $\varphi(a)=\{\nabla \in \operatorname{Pr}(L) \mid a \in \nabla\}$. Conversely, the dual of a Priestley space $\langle X, \tau, \leq\rangle$ is the distributive lattice $\left\langle\operatorname{Up}_{\tau}(X), \cap, \cup, \emptyset, X\right\rangle$, where $\operatorname{Up}_{\tau}(X)$ is the set of clopen upsets of $X$. Moreover, the function $\varphi: L \rightarrow$ $\mathrm{Up}_{\tau_{L}}(\operatorname{Pr}(L))$ is a lattice isomorphism. Thus, each distributive lattice $L$ is represented as the lattice $\operatorname{Up}_{\tau}(X)$ of clopen upsets of some Priestley space $X$. 
Let $\langle X, \tau, \leq\rangle$ be a Priestley space. If $S \subseteq X$, we write $\downarrow S=\{x \in X \mid \exists s \in S: x \leq s\}$ ( $\uparrow$ is defined dually). Following [13, Section 3], we define the operators $\mathbf{J}$ and $\mathbf{D}$ on the powerset of $X$ as follows:

$$
\begin{aligned}
\mathbf{J} S & =-\downarrow-\mathbf{I} S, \\
\mathbf{D} S & =\uparrow \mathbf{C} S .
\end{aligned}
$$

Then for each $S \subseteq X$ we have $\mathbf{J} S$ is the largest open upset contained in $S$ and $\mathbf{D} S$ is the smallest closed upset containing $S$. Now define

$$
\mathcal{R O}(X)=\{S \subseteq X \mid \mathbf{J D} S=S\}
$$

Because $\mathbf{J}$ and $\mathbf{D}$ form a Galois connection between the complete lattices of open upsets and closed upsets of $X$, we have that $\mathcal{R O}(X)$ is a complete lattice under settheoretic inclusion [13, Lemma 3.4].

Lemma 3.2 Let $L$ be a distributive lattice and let $\langle X, \tau, \leq\rangle$ be its Priestley space. Then:

(1) There is an isomorphism $f: \bar{L} \rightarrow \mathcal{R O}(X)$ such that $f \circ \eta=\varphi$. Therefore, $(\bar{L}, \eta) \simeq(\mathcal{R O}(X), \varphi)$.

(2) There is an isomorphism $g: L^{\sigma} \rightarrow \mathrm{Up}(X)$ such that $g \circ \zeta=\varphi$. Therefore, $\left(L^{\sigma}, \zeta\right) \simeq(\mathrm{Up}(X), \varphi)$.

(3) There is an isomorphism $h: \widehat{L} \rightarrow \mathrm{Up}(X)$ such that $h \circ \iota=\varphi$. Therefore, $(\widehat{L}, \iota) \simeq$ $(\mathrm{Up}(X), \varphi)$.

Proof

(1) As in [13], we view $\bar{L}$, up to isomorphism, as the lattice of normal ideals of $L$. Then $\eta: L \rightarrow \bar{L}$ is given by $\eta(a)=\downarrow a$. It was shown in [13, Theorem 3.5] that $f: \bar{L} \rightarrow \mathcal{R O}(X)$, given by $f(I)=\bigcup\{\varphi(a) \mid a \in I\}$, is a lattice isomorphism. We show that $f \circ \eta=\varphi$. For $a \in L$ we have $f(\eta(a))=f(\downarrow a)=\bigcup\{\varphi(b) \mid b \leq a\}=$ $\varphi(a)$.

(2) is well-known; see, e.g., [10, Section 2].

(3) It was shown in [3, Theorem 2.11] that $L^{\sigma}$ is isomorphic to $\widehat{L}$. Consequently, $\widehat{L}$ is isomorphic to $\operatorname{Up}(X)$. The isomorphism $h: \widehat{L} \rightarrow \mathrm{Up}(X)$ can be defined explicitly as follows. Let $\theta \in \Phi_{L}$. It is well-known that the 1-1 correspondence between congruences of $L$ and closed subsets of $X$ restricts to the 1-1 correspondence between elements of $\Phi_{L}$ and finite subsets of $X$. Let $X_{\theta}$ be the finite subset of $X$ corresponding to $\theta$. For $\alpha \in \widehat{L}$ we have $\alpha(\theta)=a / \theta$ for some $a \in L$. Then $\varphi(a) \cap X_{\theta}$ is an upset of $X_{\theta}$. If there is another $b \in L$ such that $\alpha(\theta)=b / \theta$, then $\varphi(a) \cap X_{\theta}=\varphi(b) \cap X_{\theta}$. Thus, $\varphi(a) \cap X_{\theta}$ is independent of $a \in L$, and we denote it by $\varphi(\alpha(\theta))$. Let $U_{\alpha}=\bigcup\left\{\varphi(\alpha(\theta)) \mid \theta \in \Phi_{L}\right\}$. We show that $U_{\alpha}$ is an upset of $X$. If $x \in U_{\alpha}$ and $x \leq y$, then there is $\theta \in \Phi_{L}$ such that $x \in \varphi(\alpha(\theta))$. Since $\{x, y\}$ is a finite subset of $X$, there is $\kappa \in \Phi_{L}$ such that $X_{\kappa}=\{x, y\}$. Let $\rho=\theta \cap \kappa$. Then $X_{\rho}=X_{\theta} \cup X_{\kappa}$. Therefore, $y \in X_{\rho} \subseteq U_{\alpha}$, and so $U_{\alpha} \in \operatorname{Up}(X)$. Define $h: \widehat{L} \rightarrow \operatorname{Up}(X)$ by $h(\alpha)=U_{\alpha}$. Then $h$ is the desired isomorphism. We show that $h \circ \iota=\varphi$. For $a \in L$ we have $h(\iota(a))=h\left(\alpha_{a}\right)=U_{\alpha_{a}}$. Since $U_{\alpha_{a}}=\bigcup\left\{\varphi(a) \cap X_{\theta} \mid \theta \in \Phi_{L}\right\}$, then $U_{\alpha_{a}}$ is the union of finite subsets of $\varphi(a)$. But so is $\varphi(a)$. Thus, $U_{\alpha_{a}}=\varphi(a)$, and so $h(\iota(a))=\varphi(a)$. 
We recall that a point $x$ of a topological space $X$ is isolated if $\{x\}$ is an open subset of $X$.

Definition 3.3 Let $\langle X, \tau, \leq\rangle$ be a Priestley space. We call $x \in X$ an order-isolated point if both $\uparrow x$ and $\downarrow x$ are clopen. Let $X_{0}$ denote the set of order-isolated points of $X$.

Since in a Priestley space $\langle X, \tau, \leq\rangle$ both $\uparrow x$ and $\downarrow x$ are always closed for each $x \in X$, we can equivalently define $x$ to be order-isolated if both $\uparrow x$ and $\downarrow x$ are open. Clearly every order-isolated point is isolated, but the converse is not true as follows from [2, Example 2.8].

Theorem 3.4 Let $L$ be a distributive lattice and let $X$ be its Priestley space.

(1) $(p, q)$ splits L iff there is $x \in X_{0}$ such that $\varphi(p)=\uparrow x$ and $\varphi(q)=-\downarrow x$.

(2) $p \in S(L)$ iff there is $x \in X_{0}$ such that $\varphi(p)=\uparrow x$.

(3) $q \in C S(L)$ iff there is $x \in X_{0}$ such that $\varphi(q)=-\downarrow x$.

(4) The posets $\langle S(L), \geq\rangle,\langle C S(L), \geq\rangle$, and $\left\langle X_{0}, \leq\right\rangle$ are order-isomorphic.

Proof (1) First suppose that there is $x \in X_{0}$ such that $\varphi(p)=\uparrow x$ and $\varphi(q)=-\downarrow x$. Then $\varphi(p) \nsubseteq \varphi(q)$, and so $p \not \subset q$. Therefore, $\uparrow p \cap \downarrow q=\emptyset$. Moreover, for $a \in L$ we have that either $x \in \varphi(a)$ or $x \notin \varphi(a)$. If $x \in \varphi(a)$, then $\uparrow x \subseteq \varphi(a)$, as $\varphi(a)$ is an upset of $X$. Therefore, $\varphi(p) \subseteq \varphi(a)$, and so $a \in \uparrow p$. And if $x \notin \varphi(a)$, then $\downarrow x \cap \varphi(a)=\emptyset$. Thus, $\varphi(a) \subseteq-\downarrow x$, so $\varphi(a) \subseteq \varphi(q)$, and so $a \in \downarrow q$. Consequently, $\uparrow p \cup \downarrow q=L$, and so $(p, q)$ splits $L$.

Conversely, suppose that $(p, q)$ splits $L$. Then $p \in S(L)$ and $q \in C S(L)$. Therefore, $p$ is join-prime and $q$ is meet-prime. By [2, Theorem 2.7.1], there is $x \in X$ such that $\varphi(p)=\uparrow x$. A similar argument also gives us that there is $y \in X$ such that $\varphi(q)=-\downarrow y$. We show that $x=y$. Since $p \not \subset q$, we have $\varphi(p) \nsubseteq \varphi(q)$. Therefore, $\uparrow x \nsubseteq-\downarrow y$, so $\uparrow x \cap \downarrow y \neq \varnothing$, and so $x \leq y$. If $y \nless x x$, then there is $a \in L$ such that $y \in \varphi(a)$ and $x \notin \varphi(a)$. It follows that $\uparrow x \nsubseteq \varphi(a)$, so $\varphi(p) \nsubseteq \varphi(a)$, and so $a \notin \uparrow p$. Since $(p, q)$ splits $L$, we have $a \in \downarrow q$. Therefore, $\varphi(a) \subseteq \varphi(q)=-\downarrow y$. Thus, $\varphi(a) \cap \downarrow y=\emptyset$, and so $y \notin \varphi(a)$. The obtained contradiction proves that $y \leq x$, and so $x=y$. This implies that both $\uparrow x=\varphi(p)$ and $\downarrow x=-\varphi(q)$ are clopen, which means that $x \in X_{0}$. Consequently, there is $x \in X_{0}$ such that $\varphi(p)=\uparrow x$ and $\varphi(q)=-\downarrow x$.

(2) and (3) are immediate consequences of (1).

(4) It is obvious that $\langle S(L), \geq\rangle$ is isomorphic to $\langle C S(L), \geq\rangle$ (for $p \in S(L)$, there is a unique $q \in C S(L)$ such that $(p, q)$ is a splitting pair, and sending $p$ to $q$ is the desired isomorphism). We show that $\langle C S(L), \geq\rangle$ is isomorphic to $\left\langle X_{0}, \leq\right\rangle$. Let $q \in C S(L)$. By (3), there is $x_{q} \in X_{0}$ such that $\varphi(q)=-\downarrow x_{q}$. We define $f:\langle C S(L), \geq\rangle \rightarrow\left\langle X_{0}, \leq\right\rangle$ by $f(q)=x_{q}$, and show that $f$ is an order-isomorphism. That $f$ is onto follows from (3). For $q, r \in C S(L)$, we have $q \geq r$ iff $\varphi(q) \supseteq \varphi(r)$ iff $-\downarrow x_{q} \supseteq-\downarrow x_{r}$ iff $\downarrow x_{q} \subseteq \downarrow x_{r}$ iff $x_{q} \leq x_{r}$ iff $f(q) \leq f(r)$, whence $f$ is an order-isomorphism.

Theorem 3.5 Let $L$ be a distributive lattice and let $X$ be its Priestley space. Then $L$ is principally separated iff $X_{0}$ is dense in $X$.

Proof Suppose that $L$ is principally separated and let $\varphi(a)-\varphi(b) \neq \emptyset$ be a basic open subset of $X$. From $\varphi(a)-\varphi(b) \neq \varnothing$ it follows that $a \not \leq b$. Therefore, there is a 
splitting pair $(p, q)$ such that $p \leq a$ and $b \leq q$. By Theorem 3.4.1, there is $x \in X_{0}$ such that $\varphi(p)=\uparrow x$ and $\varphi(q)=-\downarrow x$. Thus, $x \in \varphi(a)-\varphi(b)$, whence $X_{0}$ is dense in $X$. Conversely, suppose that $X_{0}$ is dense in $X$. Let $a, b \in L$ with $a\lfloor z b$. Then $\varphi(a) \nsubseteq \varphi(b)$, and so $\varphi(a)-\varphi(b)$ is a nonempty open subset of $X$. By density of $X_{0}$, there is $x \in X_{0} \cap(\varphi(a)-\varphi(b))$. Therefore, there are $p, q \in L$ such that $\varphi(p)=\uparrow x$ and $\varphi(q)=-\downarrow x$. By Theorem 3.4.1, $(p, q)$ is a splitting pair. Moreover, $\varphi(p) \subseteq \varphi(a)$ and $\varphi(b) \subseteq \varphi(q)$. Therefore, $p \leq a$ and $b \leq q$, and so $L$ is principally separated.

For a Priestley space $\langle X, \tau, \leq\rangle$, let $X_{\text {iso }}$ denote the set of isolated points of $X$.

Lemma 3.6 If $X_{0}$ is dense in $X$, then $X_{0}=X_{\text {iso. }}$.

Proof We already pointed out that $X_{0}$ is always a subset of $X_{\text {iso }}$. Suppose $X_{0}$ is dense in $X$ and $x \in X_{\text {iso }}$. Then $\{x\} \cap X_{0} \neq \emptyset$, implying that $x \in X_{0}$. Thus, $X_{\text {iso }} \subseteq X_{0}$.

Now we give necessary and sufficient conditions for the MacNeille completion of a distributive lattice to be isomorphic to the lattice of upsets of a poset. The equivalences $(2) \Leftrightarrow(3) \Leftrightarrow(7)$ can be found in [5, Sec. 4] (see also [6, Sec. 4]). Our contribution is the dual characterization of such distributive lattices. Let $L$ be a distributive lattice and let $X$ be its Priestley space. For $a \in L$ we let $\varphi_{0}(a)=$ $\varphi(a) \cap X_{0}$.

Theorem 3.7 For a distributive lattice $L$ and its Priestley space $X$, the following conditions are equivalent:

(1) $\bar{L}$ is completely join-prime generated.

(2) $\bar{L}$ is principally separated.

(3) L is principally separated.

(4) $X_{0}$ is dense in $X$.

(5) There exists an isomorphism of completions $\xi:(\bar{L}, \eta) \rightarrow\left(\mathrm{Up}\left(X_{0}\right), \varphi_{0}\right)$.

(6) $\bar{L} \simeq \mathrm{Up}\left(X_{0}\right)$.

(7) $\bar{L} \simeq \mathrm{Up}(Y)$ for some poset $Y$.

Proof The equivalence (1) $\Leftrightarrow(2)$ is obvious since $\bar{L}$ is complete; for $(2) \Leftrightarrow(3) \Leftrightarrow(7)$ see [5, Sec. 4]; for (3) $\Leftrightarrow$ (4) see Theorem 3.5; and (5) $\Rightarrow(6) \Rightarrow(7)$ are immediate. Thus, to complete the proof, we need to show that (4) $\Rightarrow(5)$.

$(4) \Rightarrow(5)$ : We define $\varepsilon: \mathcal{R O}(X) \rightarrow \mathrm{Up}\left(X_{0}\right)$ by $\varepsilon(U)=U \cap X_{0}$. This function is well-defined because every $U \in \mathcal{R O}(X)$ is an upset. We show that $\varepsilon$ is an orderisomorphism. For $U, V \in \mathcal{R O}(X)$ with $U \subseteq V$ we have $\varepsilon(U)=U \cap X_{0} \subseteq V \cap X_{0}=$ $\varepsilon(V)$. Suppose that $U \nsubseteq V$. We show that $U \nsubseteq \mathbf{D} V$. If $U \subseteq \mathbf{D} V$, then $U \cap-\mathbf{D} V=\emptyset$. Since $U$ is an upset, this implies $U \cap \downarrow-\mathbf{D} V=\emptyset$. Therefore, as $\mathbf{J D} V=V$, we obtain $U \subseteq-\downarrow-\mathbf{D} V=\mathbf{J D} V=V$, a contradiction. Thus, $U \nsubseteq \mathbf{D} V$, and so $U-\mathbf{D} V$ is a nonempty open subset of $X$. Because $X_{0}$ is dense in $X$, there exists $x \in X_{0} \cap(U-$ D $V)$. This implies that $x \in U \cap X_{0}$ and $x \notin V \cap X_{0}$, and so $\varepsilon(U) \nsubseteq \varepsilon(V)$. Consequently, $U \subseteq V$ iff $\varepsilon(U) \subseteq \varepsilon(V)$. To see that $\varepsilon$ is onto, let $U \in \operatorname{Up}\left(X_{0}\right)$. We show that $\varepsilon(\mathbf{J D} U)=U$. Clearly $U \subseteq \mathbf{J D} U \cap X_{0}=\varepsilon(\mathbf{J D} U)$. For the converse inclusion, suppose that $x \in \varepsilon(\mathbf{J D} U)=\mathbf{J D} U \cap X_{0}$. Then $x \in-\downarrow-$ ID $U$, whence $x \notin \downarrow-$ ID $U$. It follows that $\uparrow x \cap-\mathbf{I D} U=\emptyset$. So $\uparrow x \subseteq \mathbf{I D} U \subseteq \mathbf{D} U=\uparrow \mathbf{C} U$. Therefore, $x \in \uparrow \mathbf{C} U$, and so $\downarrow x \cap \mathbf{C} U \neq \emptyset$. Since $x \in X_{0}$, then $\downarrow x$ is open. Thus, $\downarrow x \cap U \neq \emptyset$, and as $U$ 
is an upset of $X_{0}$, it follows that $x \in U$. This proves that $\mathbf{J D} U \cap X_{0} \subseteq U$, whence $\varepsilon(\mathbf{J D} U)=U$. Consequently, $\varepsilon$ is an isomorphism from $\mathcal{R O}(X)$ onto $\operatorname{Up}\left(X_{0}\right)$. By Lemma 3.2.1, there is an isomorphism $f: \bar{L} \rightarrow \mathcal{R O}(X)$ such that $f \circ \eta=\varphi$. We set $\xi=\varepsilon \circ f$. Then $\xi \circ \eta=\varepsilon \circ f \circ \eta=\varepsilon \circ \varphi=\varphi_{0}$. Thus, there is an isomorphism $\xi: \bar{L} \rightarrow \mathrm{Up}\left(X_{0}\right)$ such that $\xi \circ \eta=\varphi_{0}$.

Remark 3.8 It follows that if a distributive lattice is principally separated, then its MacNeille completion is distributive (even completely distributive) [5, Sec. 4], thus providing a sufficient condition for the MacNeille completion of a distributive lattice to be distributive. (In fact, each principally separated lattice is distributive. Therefore, that $\bar{L}$ is completely distributive already follows from $L$ being principally separated.)

Let $L$ be a distributive lattice. If $L$ is principally separated, then we saw that $\bar{L}$ is isomorphic to $\operatorname{Up}(Y)$ for some poset $Y$. But this does not mean that $\bar{L}$ is isomorphic to the lattice of upsets of the Priestley space of $L$, as the following example shows.

Example 3.9 Let $L$ be the negative integers with bottom, and let $X$ be its Priestley space. Then $\langle X, \leq\rangle$ is isomorphic to the positive integers with top, $L$ is complete and principally separated, so $\bar{L} \simeq L$, but $L \not \operatorname{Up}(X)$ (see Fig. 2). Since $\operatorname{Up}(X)$ is isomorphic to $L^{\sigma}$, it follows that $L^{\sigma} \not \subset \bar{L}$.

Theorem 3.10 Let $L$ be a distributive lattice and let $X$ be its Priestley space. Then $\bar{L}$ is isomorphic to $L^{\sigma}$ iff $X_{0}$ is dense in $X$ and $\langle X, \leq\rangle$ is order-isomorphic to $\left\langle X_{0}, \leq\right\rangle$.

Proof First suppose that $X_{0}$ is dense in $X$ and $\langle X, \leq\rangle$ is order-isomorphic to $\left\langle X_{0}, \leq\right\rangle$. Then $\operatorname{Up}\left(X_{0}\right) \simeq \operatorname{Up}(X)$. By Theorem 3.7 and Lemma 3.2, $\bar{L} \simeq \operatorname{Up}\left(X_{0}\right)$ and $L^{\sigma} \simeq$ $\mathrm{Up}(X)$. Thus, $\bar{L} \simeq L^{\sigma}$. Now suppose that $\bar{L} \simeq L^{\sigma}$. Then $\bar{L}$ is completely join-prime generated, and by Theorem 3.7, $X_{0}$ is dense in $X$. Moreover, since $\bar{L} \simeq \mathrm{Up}\left(X_{0}\right)$ and $L^{\sigma} \simeq \mathrm{Up}(X)$, from $\bar{L} \simeq L^{\sigma}$ it follows that $\mathrm{Up}\left(X_{0}\right) \simeq \mathrm{Up}(X)$. This by the well-known duality between complete and completely join-prime generated distributive lattices and partially ordered sets implies that $\left\langle X_{0}, \leq\right\rangle$ is order-isomorphic to $\langle X, \leq\rangle$.

Fig. 2 Example 3.9
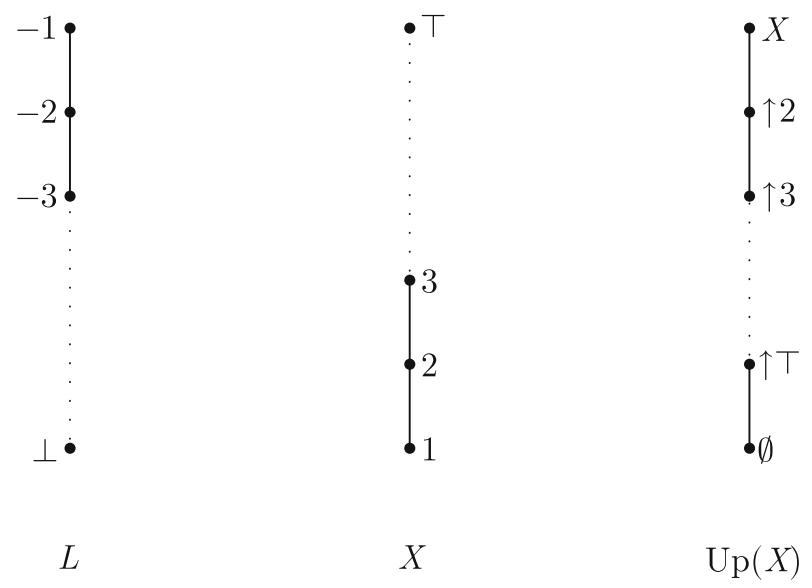


$\begin{array}{lll}\circ & \circ & \circ \\ 0 & 1 & 2\end{array}$

Fig. 3 Example 3.12(1)

Corollary 3.11 Let $L$ be a distributive lattice and let $X$ be its Priestley space. Then the following conditions are equivalent:

(1) The three completions $\bar{L}, L^{\sigma}$, and $\widehat{L}$ of $L$ are isomorphic.

(2) $X_{0}$ is dense in $X$ and $\langle X, \leq\rangle$ is order-isomorphic to $\left\langle X_{0}, \leq\right\rangle$.

(3) $L$ is principally separated and the poset $\langle S(L), \geq\rangle$ is order-isomorphic to the poset of prime filters of $L$.

Proof By Lemma 3.2, $L^{\sigma} \simeq \widehat{L}$. Therefore, the equivalence of (1) and (2) follows from Theorem 3.10. That (2) is equivalent to (3) is a consequence of Theorems 3.5 and 3.4.

Example 3.12 To show the utility of Corollary 3.11, we give several concrete examples.

(1) Let $B$ denote the Boolean lattice of finite and cofinite subsets of $\omega$. It is wellknown that the dual space $X$ of $B$ is the one-point compactification of $\omega$, which is homeomorphic to $\omega+1$ in its interval topology (see Fig. 3). Therefore, $X_{0}=X_{\text {iso }}=\omega$, and so $X_{0}$ is dense in $X$. Moreover, because both $\omega$ and $X$ are countable, and $\leq$ is simply the equality relation, we obtain that $\langle X, \leq\rangle$ is order-isomorphic to $\left\langle X_{0}, \leq\right\rangle$. Consequently, by Corollary $3.11, \bar{B}, B^{\sigma}$, and $\widehat{B}$ are isomorphic. Note that $(\bar{B}, \eta),\left(B^{\sigma}, \zeta\right)$, and $(\widehat{B}, \iota)$ cannot be isomorphic as completions because $B$ is an infinite Boolean algebra (see Theorem 4.8).

(2) Next, consider the Priestley space $\langle X, \tau, \leq\rangle$ shown in Fig. 4, where each $(i, j)$ is isolated and each $(\omega, j)$ is a limit point of $X(i \in \omega$ and $0 \leq j \leq n)$. Clearly $X_{0}=$ $\{(i, j) \mid i \in \omega$ and $0 \leq j \leq n\}$ and $X$ is the $n$-point compactification of the discrete space $X_{0}$. Of course, $X$ is a finite disjoint union of the spaces $X^{i}=\{(n, i) \mid$
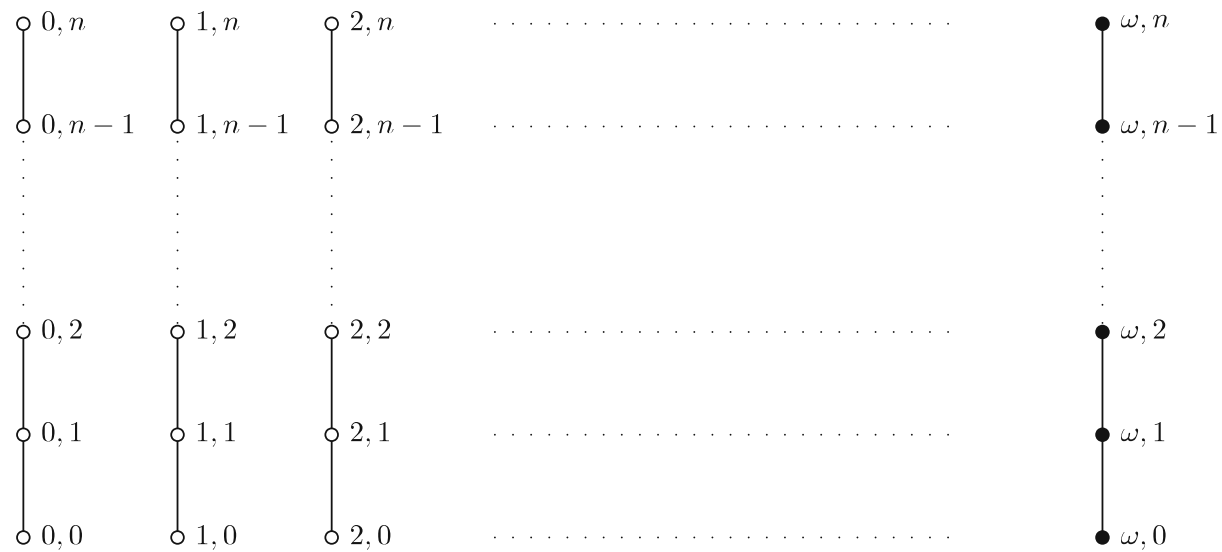

Fig. 4 Example 3.12(2) 
$n \in \omega\} \cup\{(\omega, i)\}, 0 \leq i \leq n$, where each $X^{i}$ is the one-point compactification of the discrete space $\{(n, i) \mid n \in \omega\}$. We can view this as a generalization of the previous example: we simply replace each point of the previous example by an $(n+1)$-point chain. It follows from the definition that $X_{0}$ is dense in $X$, and it is easy to see that $\langle X, \leq\rangle$ is order-isomorphic to $\left\langle X_{0}, \leq\right\rangle$. Let $L$ denote the lattice of clopen upsets of $X$. Then, by Corollary $3.11, \bar{L}, L^{\sigma}$, and $\widehat{L}$ are isomorphic. By Theorem 3.14, $(\bar{L}, \eta),\left(L^{\sigma}, \zeta\right)$, and $(\widehat{L}, \iota)$ cannot be isomorphic as completions of $L$.

(3) Finally, consider the Priestley space $\langle X, \tau, \leq\rangle$ shown in Fig. 5, where each of $(i, j)$ is isolated, and each of $(\omega, j),(i, \omega)$, and $(\omega, \omega)$ is a limit point of $X$ $(i, j \in \omega)$. Clearly $X_{0}=\{(i, j) \mid i, j \in \omega\}$ and $X$ is a countable compactification of the discrete space $X_{0}$. We can think of each $X^{i}=\{(0, i),(1, i), \ldots,(\omega, i)\} \cup$ $\{(i, 0),(i, 1) \ldots,(i, \omega)\}$ as the two-point compactification of the discrete space $\{(0, i),(1, i), \ldots\} \cup\{(i, 0),(i, 1), \ldots\}$, and of $X$ as the one-point compactification of $X-\{(\omega, \omega)\}$ (which is not a discrete space). Therefore, we can view this as a generalization of the previous example: we replace each $(n+1)$-point chain by an $(\omega+1)$-point chain. It is obvious that $X_{0}$ is dense in $X$. However, $\langle X, \leq\rangle$ is not order-isomorphic to $\left\langle X_{0}, \leq\right\rangle$. Let $L$ denote the lattice of clopen upsets of $X$. Then it follows from Corollary 3.11 that $\bar{L}$ is not isomorphic to neither $L^{\sigma}$ nor $\widehat{L}$.

Based on Example 3.12, it is tempting to conjecture that the MacNeille completion $\bar{L}$ of a distributive lattice $L$ is isomorphic to $L^{\sigma}$ and $\widehat{L}$ only if the Priestley space of $L$ does not have any infinite chains. This, however, is not the case as we show in the next example.

Example 3.13 Consider the Priestley space $\langle X, \tau, \leq\rangle$ shown in Fig. 6, where $\{(\omega, n) \mid$ $n \in \omega\} \cup\{(n, \omega) \mid n \in \omega\} \cup\{(\omega, \omega)\}$ are the limit points of $X$, and the rest are isolated in $X$. Clearly $X_{0}=X-(\{(\omega, n) \mid n \in \omega\} \cup\{(n, \omega) \mid n \in \omega\} \cup\{(\omega, \omega)\})$ and $X$ is a countable compactification of the discrete space $X_{0}$. We can think of

$\bullet 0, \omega$
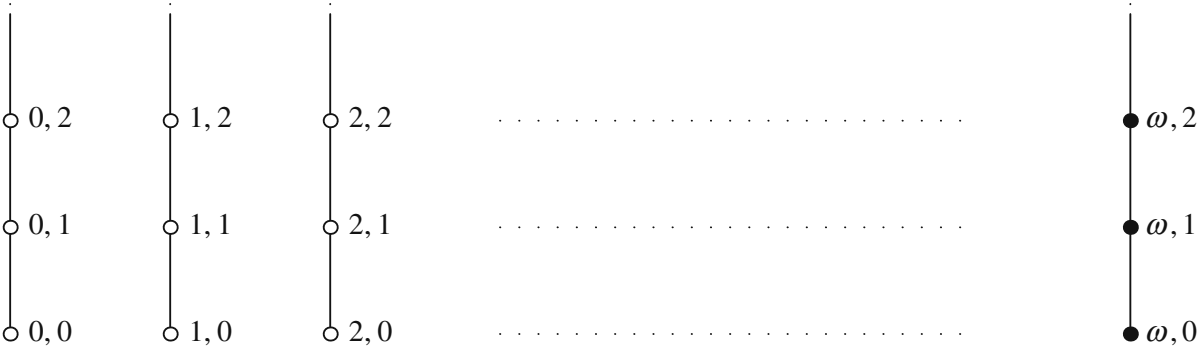

Fig. 5 Example 3.12(3) 

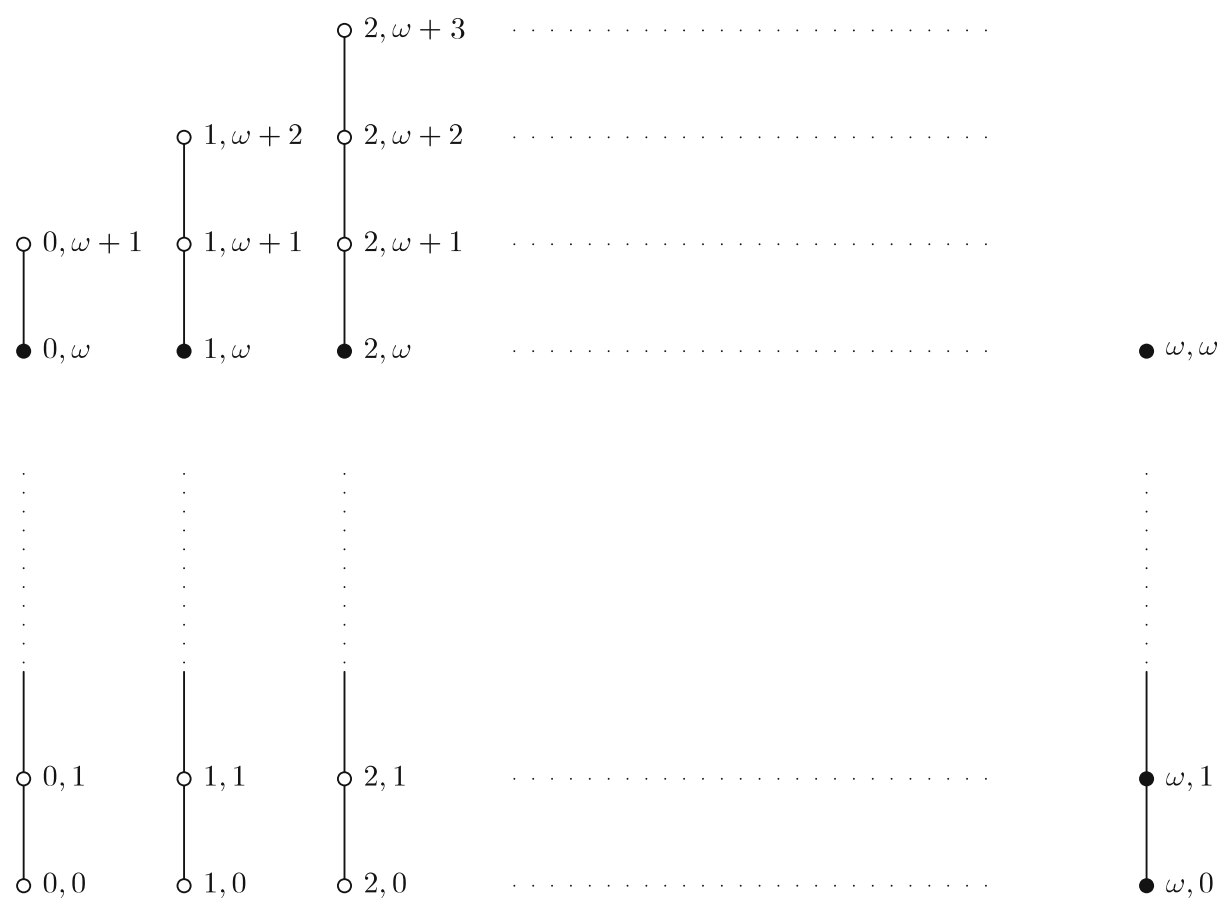

Fig. 6 Example 3.13

each $X^{i}=\{(0, i),(1, i), \ldots,(\omega, i)\} \cup\{(i, 0),(i, 1) \ldots,(i, \omega),(i, \omega+1, \ldots,(i, \omega+i+1))\}$ as the two-point compactification of the discrete space $X^{i}-\{(\omega, i),(i, \omega)\}$, and of $X$ as the one point compactification of $X-\{(\omega, \omega)\}$ (which is not a discrete space). Therefore, we can view this as a further generalization of the spaces in Example 3.12. Clearly $X$ has (infinitely many) infinite chains. It is also obvious that $X_{0}$ is dense in $X$. We show that there is an order-isomorphism from $X_{0}$ onto $X$. Define $f: X_{0} \rightarrow X$ as follows:

$$
f:(\alpha, \beta) \mapsto \begin{cases}(\omega, \beta) & \text { if } \alpha=0, \beta<\omega, \\ (\omega, \omega) & \text { if } \alpha=0, \beta=\omega+1, \\ (\alpha-1, \beta) & \text { if } \alpha>0, \beta<\omega, \\ (\alpha-1, \beta-1) & \text { if } \alpha>0, \beta>\omega .\end{cases}
$$

The map $f$ is shown in Fig. 7, from which it is easy to see that $f$ is an orderisomorphism. Let $L$ be the lattice of clopen upsets of $X$. Then, by Corollary 3.11, $\bar{L} \simeq L^{\sigma}$, in spite of the fact that $X$ has infinite ascending chains. By taking the opposite of $X$, we obtain an example of a Priestley space $X$ with infinitely descending chains such that $X_{0}$ is order-isomorphic to $X$. Thus, there exist distributive lattices $L$ whose Priestley spaces have both infinitely ascending and descending chains, but still $\bar{L}$ is isomorphic to $L^{\sigma}$ (and hence to $\widehat{L}$ ).

The final goal of this section is to investigate when $(\bar{L}, \eta),\left(L^{\sigma}, \zeta\right)$, and $(\widehat{L}, \iota)$ are isomorphic as completions of $L$. 

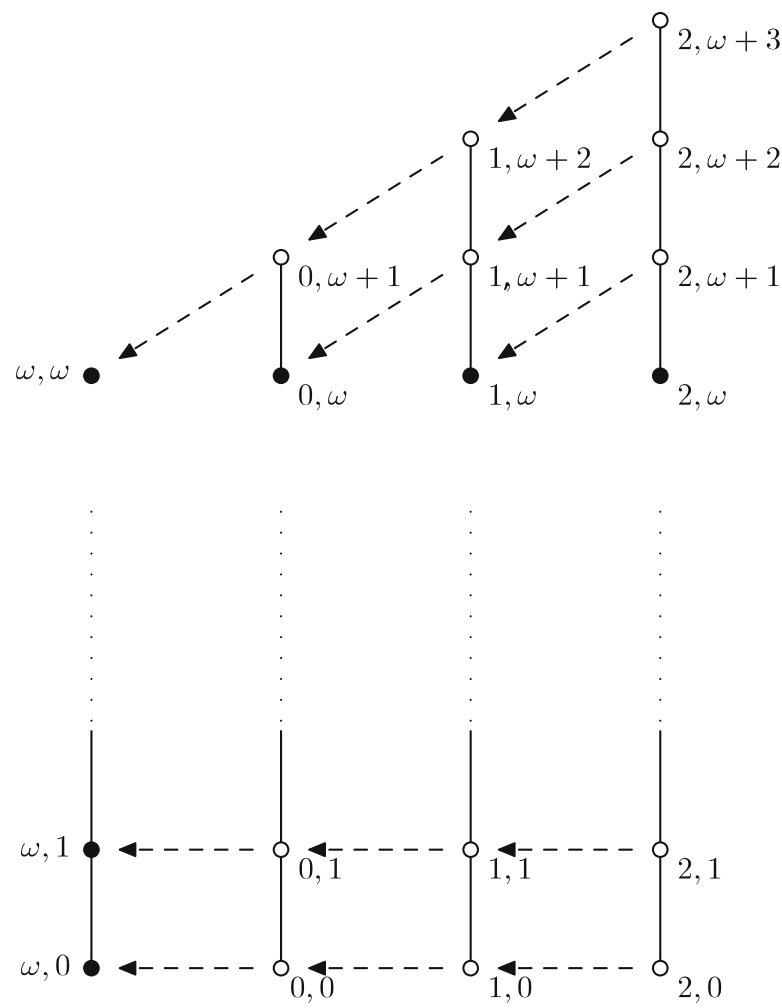

Fig. 7 The map $f: X_{0} \rightarrow X$

Theorem 3.14 Let $L$ be a distributive lattice. The three completions $(\bar{L}, \eta),\left(L^{\sigma}, \zeta\right)$, and $(\widehat{L}, \iota)$ are isomorphic as completions of $L$ iff $L$ is finite.

Proof By Lemma 3.2, it is sufficient to show that there is an isomorphism $\alpha: \bar{L} \rightarrow L^{\sigma}$ such that $\alpha \circ \eta=\zeta$ iff $L$ is finite. Clearly if $L$ is finite, then $\eta$ and $\zeta$ are identities. Therefore, if we let $\alpha$ be the identity, then $\alpha \circ \eta=\zeta$. Conversely, suppose that there exists $\alpha: \bar{L} \rightarrow L^{\sigma}$ such that $\alpha \circ \eta=\zeta$. Since $\bar{L} \simeq L^{\sigma}$, then $\bar{L}$ is completely joinprime generated, and by Theorem 3.7, $X_{0}$ is dense in $X$. We show that $X_{0}=X$. By Lemma 3.2, $(\bar{L}, \eta) \simeq\left(\mathrm{Up}\left(X_{0}\right), \varphi_{0}\right)$ and $\left(L^{\sigma}, \zeta\right) \simeq(\mathrm{Up}(X), \varphi)$. Therefore, $\alpha \circ \eta=\zeta$ implies there is a lattice isomorphism $\lambda: \operatorname{Up}\left(X_{0}\right) \rightarrow \operatorname{Up}(X)$ such that $\lambda \circ \varphi_{0}=\varphi$. Let $x \in X$. Then $\uparrow x \in \operatorname{Up}(X)$. Since $\lambda$ is onto, there is $U \in \operatorname{Up}\left(X_{0}\right)$ such that $\lambda(U)=\uparrow x$. We have $U=\bigcup\left\{\uparrow y \cap X_{0} \mid y \in U\right\}$. From $y \in X_{0}$ it follows that $\uparrow y$ is a clopen upset of $X$. Therefore, there is $a_{y} \in L$ such that $\uparrow y=\varphi\left(a_{y}\right)$. Thus,

$$
\begin{aligned}
U & =\bigcup\left\{\uparrow y \cap X_{0} \mid y \in U\right\} \\
& =\bigcup\left\{\varphi\left(a_{y}\right) \cap X_{0} \mid y \in U\right\} \\
& =\bigcup\left\{\varphi_{0}\left(a_{y}\right) \mid y \in U\right\},
\end{aligned}
$$


and so

$$
\begin{aligned}
\uparrow x & =\lambda(U) \\
& =\lambda\left(\bigcup\left\{\varphi_{0}\left(a_{y}\right) \mid y \in U\right\}\right) \\
& \left.=\bigcup\left\{\lambda \varphi_{0}\left(a_{y}\right) \mid y \in U\right\}\right) \\
& \left.=\bigcup\left\{\varphi\left(a_{y}\right) \mid y \in U\right\}\right) .
\end{aligned}
$$

Consequently, there is $y \in X_{0}$ such that $\uparrow x=\varphi\left(a_{y}\right)=\uparrow y$. It follows that $x=y$, and so $x \in X_{0}$. This implies that $X=X_{0}$, which together with Lemma 3.6 give us $X_{\text {iso }}=$ $X$. Therefore, $X$ is discrete, hence finite by compactness. Thus, $L$ is finite.

We summarize matters below.

Corollary 3.15 For a distributive lattice $L$, the three lattices $\bar{L}, L^{\sigma}$, and $\widehat{L}$ are isomorphic iff $L$ is principally separated and the poset $\langle S(L), \geq\rangle$ is order-isomorphic to the poset of prime filters of $L$. Moreover, the completions $(\bar{L}, \eta),\left(L^{\sigma}, \zeta\right)$, and $(\widehat{L}, \iota)$ are isomorphic as completions of $L$ iff $L$ is finite.

\section{Heyting Algebras}

Recall that a distributive lattice $L$ is a Heyting algebra if there exists a binary operation $\rightarrow: L^{2} \rightarrow L$ such that for all $a, b, c \in L$ we have:

$$
a \wedge c \leq b \text { iff } c \leq a \rightarrow b .
$$

The duality theory for Heyting algebras was developed by Esakia [7]. As we will see shortly, the MacNeille and canonical completions of a Heyting algebra have the same dual characterization as for distributive lattices. Therefore, our results about the isomorphism of $\bar{L}$ and $L^{\sigma}$ apply unchanged for Heyting algebras. Some simplifications are possible though, because for a Heyting algebra $A$ we have $S(A)=$ $J^{\infty}(A)$. On the other hand, the dual characterization of the profinite completion of a Heyting algebra is different from that of a distributive lattice. In this section we will adjust the results of the previous section appropriately to obtain necessary and sufficient conditions for the MacNeille, canonical, and profinite completions of a Heyting algebra to be isomorphic. As a corollary, we also obtain necessary and sufficient conditions for the three completions of a Boolean algebra to be isomorphic.

Definition 4.1 [7] A triple $\langle X, \tau, \leq\rangle$ is an Esakia space if $\langle X, \tau, \leq\rangle$ is a Priestley space and $\downarrow U$ is open for each open subset $U$ of $X$.

The same way distributive lattices are represented as clopen upsets of Priestley spaces, Heyting algebras are represented as clopen upsets of Esakia spaces. 
We recall that there is a 1-1 correspondence between congruences and filters of a Heyting algebra $A$ (see, e.g., [19, Section I.13]), and that it is obtained by associating with each congruence $\theta$ of $A$, the filter $F_{\theta}=1 / \theta=\{a \in A \mid a \theta 1\}$, and with each filter $F$ of $A$, the congruence $\theta_{F}=\left\{(a, b) \in A^{2} \mid(a \rightarrow b) \wedge(b \rightarrow a) \in F\right\}$. We say that a filter $F$ of $A$ is of finite index if the quotient algebra $A / \theta_{F}$ is finite.

Let $A$ be a Heyting algebra and let $X$ be its Esakia space. We recall that filters of $A$ correspond to closed upsets of $X$ (see, e.g., [7]), and that this correspondence is obtained by associating with each filter $F$ of $A$, the closed upset $C_{F}=\bigcap\{\varphi(a) \mid a \in$ $F\}$ of $X$, and with each closed upset $C$ of $X$, the filter $F_{C}=\{a \in A \mid C \subseteq \varphi(a)\}$ of $A$. Now, if $F$ is a principal filter $\uparrow a$ of $A$, then $C_{F}=\varphi(a)$ is a clopen upset of $X$, and if $C$ is a clopen upset of $X$, then $C=\varphi(a)$ for some $a \in A$, and so $F_{C}$ is the principal filter $\uparrow a$ of $A$. Thus, principal filters of $A$ correspond to clopen upsets of $X$. Also, if $F$ is of finite index, then $C_{F}$ is a finite upset of $X$, and if $C$ is a finite upset of $X$, then $F_{C}$ is of finite index. Thus, filters of $A$ of finite index correspond to finite upsets of $X$.

As we pointed out above, in a Heyting algebra $A$ we have $S(A)=J^{\infty}(A)$, and so for Heyting algebras principally separated simply means completely join-prime generated. This follows from [2, Theorem 2.7] and the dual characterization of splitting pairs of a distributive lattice given in Theorem 3.4. We also give a simple algebraic proof. Let $a \in J^{\infty}(A)$. If $\downarrow a-\{a\}$ does not have a greatest element, then $a=\bigvee(\downarrow a-\{a\})$, which contradicts to $a \in J^{\infty}(A)$. Let $b$ be the greatest element of $\downarrow a-\{a\}$. Then $(a, a \rightarrow b)$ splits $A$. Indeed, for $c \in A$, if $a \not \leq c$, then $a \wedge c \leq b$, so $c \leq a \rightarrow b$, and so $a \in S(A)$.

Let $X$ be an Esakia space. Since for $x \in X$ we have $x \in X_{\text {iso }}$ implies $\downarrow x$ is clopen, we obtain that order-isolated points of $X$ are exactly those isolated points $x$ of $X$ for which $\uparrow x$ is (cl)open. We define

$$
X_{\text {fin }}=\{x \in X \mid \uparrow x \text { is finite }\} .
$$

In other words, $X_{\mathrm{fin}}$ is the union of all finite upsets of $X$. Let $\varphi_{\mathrm{fin}}: A \rightarrow \operatorname{Up}\left(X_{\mathrm{fin}}\right)$ be given by $\varphi_{\text {fin }}(a)=\varphi(a) \cap X_{\text {fin }}$. Then $\varphi_{\text {fin }}$ is a lattice homomorphism, and $\varphi_{\text {fin }}$ is an embedding iff $X_{\text {fin }}$ is dense in $X$ [2, Theorem 3.1 and Proposition 3.2].

Lemma 4.2 Let $A$ be a Heyting algebra and let $X$ be its Esakia space. Then:

(1) There is an isomorphism $f: \bar{A} \rightarrow \mathcal{R O}(X)$ such that $f \circ \eta=\varphi$. Therefore, $(\bar{A}, \eta) \simeq(\mathcal{R O}(X), \varphi)$. Moreover, $(\bar{A}, \eta) \simeq\left(\mathrm{Up}\left(X_{0}\right), \varphi_{0}\right)$ iff $A$ is completely join-prime generated.

(2) There is an isomorphism $g: A^{\sigma} \rightarrow \mathrm{Up}(X)$ such that $g \circ \zeta=\varphi$. Therefore, $\left(A^{\sigma}, \zeta\right) \simeq(\mathrm{Up}(X), \varphi)$.

(3) There is an isomorphism $h: \widehat{A} \rightarrow \mathrm{Up}\left(X_{\mathrm{fin}}\right)$ such that $h \circ \iota=\varphi_{\mathrm{fin}}$. Therefore, if $\iota: A \rightarrow \widehat{A}$ is an embedding, then $(\widehat{A}, \iota) \simeq\left(\mathrm{Up}\left(X_{\text {fin }}\right), \varphi_{\text {fin }}\right)$.

Proof Since $A$ is principally separated iff $A$ is completely join-prime generated, (1) follows from Lemma 3.2.1 and Theorem 3.7. Obviously (2) is a consequence of Lemma 3.2.2. To see (3), it was shown in [3, Theorem 4.7] that $\widehat{A} \simeq \mathrm{Up}\left(X_{\text {fin }}\right)$. We recall that the isomorphism $h: \widehat{A} \rightarrow \mathrm{Up}\left(X_{\text {fin }}\right)$ is explicitly defined as follows. Let $\Psi_{A}$ 
denote the set of filters of $A$ of finite index. For $F \in \Psi_{A}$, let $C_{F}$ be the finite upset of $X$ corresponding to $F$. For $\alpha \in \widehat{A}$ and $F \in \Psi_{A}$, we have $\alpha(F)=a / \theta_{F}$ for some $a \in A$. Then $\varphi(a) \cap C_{F}$ is an upset of $C_{F}$. If there is another $b \in L$ such that $\alpha(F)=b / \theta_{F}$, then $\varphi(a) \cap C_{F}=\varphi(b) \cap C_{F}$. Thus, $\varphi(a) \cap C_{F}$ is independent of $a \in L$, and we denote it by $\varphi(\alpha(F))$. Let $U_{\alpha}=\bigcup\left\{\varphi(\alpha(F)) \mid F \in \Psi_{A}\right\}$. Since each $C_{F}$ is a finite upset of $X$ and $\varphi(\alpha(F))$ is an upset of $C_{F}$, it follows that $U_{\alpha}$ is an upset of $X_{\text {fin }}$. We define $h: \widehat{A} \rightarrow$ $\mathrm{Up}\left(X_{\text {fin }}\right)$ by $h(\alpha)=U_{\alpha}$. Then $h$ is the desired isomorphism. We show that $h \circ \iota=\varphi_{\text {fin }}$. For $a \in A$ we have $h(\iota(a))=h\left(\alpha_{a}\right)=\bigcup\left\{\varphi(a) \cap C_{F} \mid F \in \Psi_{A}\right\}=\varphi(a) \cap \bigcup\left\{C_{F} \mid F \in\right.$ $\left.\Psi_{A}\right\}=\varphi(a) \cap X_{\text {fin }}=\varphi_{\text {fin }}(a)$. Here we use that $X_{\text {fin }}=\bigcup\left\{C_{F} \mid F \in \Psi_{A}\right\}$, which is true because $X_{\text {fin }}$ is the union of all finite upsets of $X$ and each finite upset of $X$ has the form $C_{F}$ for some $F \in \Psi_{A}$.

From Theorem 3.10 and Lemma 4.2 we immediately obtain:

Theorem 4.3 Let $A$ be a Heyting algebra and let $X$ be its Esakia space. Then the following conditions are equivalent:

(1) $\bar{A} \simeq A^{\sigma}$.

(2) $X_{0}$ is dense in $X$ and $\left\langle X_{0}, \leq\right\rangle$ is order-isomorphic to $\langle X, \leq\rangle$.

(3) $A$ is completely join-prime generated and $\left\langle J^{\infty}(A), \geq\right\rangle$ is order-isomorphic to the poset of prime filters of $A$.

Since the profinite completion of a Heyting algebra behaves differently from the profinite completion of a distributive lattice, the comparison of the MacNeille and canonical completions of a Heyting algebra to its profinite completion is slightly different.

Theorem 4.4 Let $A$ be a Heyting algebra and let $X$ be its Esakia space. Then:

(1) The following conditions are equivalent:

(a) $\bar{A} \simeq \widehat{A}$.

(b) $X_{0}$ is dense in $X$ and $\left\langle X_{0}, \leq\right\rangle$ is order-isomorphic to $\left\langle X_{\mathrm{fin}}, \leq\right\rangle$.

(c) $A$ is completely join-prime generated and $\left\langle J^{\infty}(A), \geq\right\rangle$ is order-isomorphic to the poset of prime filters of $A$ of finite index.

(2) The following conditions are equivalent:

(a) $A^{\sigma} \simeq \widehat{A}$.

(b) $\left(A^{\sigma}, \zeta\right) \simeq(\widehat{A}, \iota)$.

(c) $\left\langle X_{\mathrm{fin}}, \leq\right\rangle$ is order-isomorphic to $\langle X, \leq\rangle$.

(d) $\quad X_{\text {fin }}=X$.

Proof The proof of (1) is analogous to that of Theorem 3.10. For (2), note that the equivalences (a) $\Leftrightarrow$ (c) $\Leftrightarrow$ (d) follow from [3, Theorem 4.10]. Furthermore, it is clear that (b) implies (a). Finally, (d) implies (b) because from $X_{\text {fin }}=X$ it follows that $\operatorname{Up}\left(X_{\mathrm{fin}}\right)=\mathrm{Up}(X)$ and the identity $\operatorname{id}_{\mathrm{Up}(X)}: \operatorname{Up}(X) \rightarrow \mathrm{Up}(X)$ is always an isomorphism of completions. 
Putting Theorems 4.3 and 4.4 together, we obtain:

Corollary 4.5 Let $A$ be a Heyting algebra and let $X$ be its Esakia space. Then the following conditions are equivalent:

(1) $\bar{A} \simeq A^{\sigma} \simeq \widehat{A}$.

(2) $X_{0}$ is dense in $X$ and $\left\langle X_{0}, \leq\right\rangle$ is order-isomorphic to $\left\langle X_{\mathrm{fin}}, \leq\right\rangle=\langle X, \leq\rangle$.

(3) $A$ is completely join-prime generated, $\left\langle J^{\infty}(A), \geq\right\rangle$ is isomorphic to the poset of prime filters of $A$, and every prime filter of $A$ is of finite index.

We mention some of the consequences of these results below. We say that a Heyting algebra $A$ is finitely approximable, or residually finite, if $a \not \leq b$ implies there is a filter $F$ of $A$ of finite index such that $a \in F$ and $b \notin F$.

Corollary 4.6 Let $A$ be a Heyting algebra and let $X$ be its Esakia space.

(1) If the variety generated by $A$ is finitely generated, then $\bar{A} \simeq A^{\sigma} \simeq \widehat{A}$ iff $X_{0}$ is dense in $X$ and $\left\langle X_{0}, \leq\right\rangle$ is order-isomorphic to $\langle X, \leq\rangle$.

(2) If $X$ has infinite chains, then $A^{\sigma}$ is not isomorphic to $\widehat{A}$. Nevertheless, there are Heyting algebras whose Esakia spaces have infinite chains but $\bar{A}$ is still isomorphic to $A^{\sigma}$; also, there are Heyting algebras whose Esakia spaces have infinite chains but $\bar{A}$ is still isomorphic to $\widehat{A}$.

(3) If $A$ is a finitely generated and finitely approximable Heyting algebra, then $\bar{A}$ is isomorphic to $\widehat{A}$.

Proof (1) follows from Corollary 4.5 and the fact that $X_{\text {fin }}=X$ whenever $A$ generates a finitely generated variety of Heyting algebras (see, e.g., [3, Theorem 5.1]). For (2), observe that if $X$ has infinite chains, then $X_{\text {fin }} \neq X$, and apply Theorem 4.4.2. For an example of a Heyting algebra $A$ whose Esakia space has infinite chains but $\bar{A}$ is still isomorphic to $A^{\sigma}$, observe that the Priestley space constructed in Example 3.13 is in fact an Esakia space. Therefore, the corresponding Heyting algebra is our desired example. Finally, for an example of a Heyting algebra $A$ whose Esakia space has infinite chains but $\bar{A}$ is still isomorphic to $\widehat{A}$ take the Heyting algebra $A$ of positive integers with top. The Esakia space $X$ of $A$ is order-isomorphic to the negative integers with bottom (see Fig. 8). So $X_{0}=X_{\text {fin }}$, and so $\bar{A} \simeq \widehat{A} \simeq A$. For (3) observe

Fig. 8 Corollary 4.6 .2
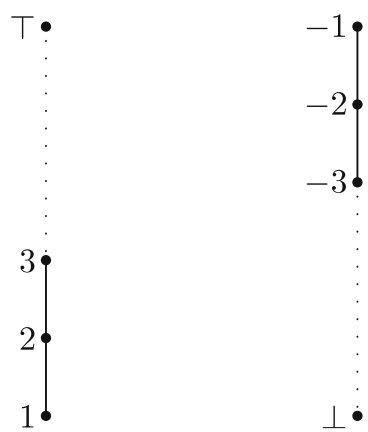
that if $A$ is a finitely generated and finitely approximable Heyting algebra, then it follows from [4, Section 3.1] and [2, Theorem 3.1] that $X_{0}=X_{\text {iso }}=X_{\text {fin }}$ and that $X_{0}$ is dense in $X$, thus the result follows from Theorem 4.4.1.

Corollary 4.7 For a Boolean algebra A, the following conditions are equivalent:

(1) $\bar{A} \simeq A^{\sigma} \simeq \widehat{A}$.

(2) $A$ is atomic and the set of atoms of $A$ has the same cardinality as the set of ultrafilters of $A$.

(3) $A$ is atomic and the cardinality of the set of free ultrafilters of $A$ is less than or equal to the cardinality of the set of atoms of $A$.

Proof Suppose $A$ is a Boolean algebra and $X$ is its Stone space. Then $X_{0}=X_{\text {iso }}$, there is a bijection between atoms of $A$ and $X_{\text {iso }}$, and $A$ is atomic iff $X_{\text {iso }}$ is dense in $X$. Moreover, an order-isomorphism between $X_{0}$ and $X$ is simply a bijection. Now since the variety of Boolean algebras is a finitely generated variety of Heyting algebras, the result follows from Corollary 4.6.1.

The rest of this section we will be concerned with necessary and sufficient conditions for the MacNeille, canonical, and profinite completions of a Heyting algebra $A$ to be isomorphic as completions of $A$. First off, as a reformulation of Theorem 3.14 we obtain:

Theorem 4.8 For a Heyting algebra A, there is an isomorphism of completions $\alpha:(\bar{A}, \eta) \rightarrow\left(A^{\sigma}, \zeta\right)$ iff $A$ is finite. In particular, if $A$ is a Boolean algebra, then $(\bar{A}, \eta) \simeq\left(A^{\sigma}, \zeta\right)$ iff $A$ is finite.

Now we obtain a necessary and sufficient condition for an isomorphism $\beta: \bar{A} \rightarrow$ $\widehat{A}$ to commute with $\eta$ and $\iota$.

Lemma 4.9 Let $\langle X, \tau\rangle$ be a topological space with $Y \subseteq X$ dense in $X$. If $Z \subseteq X$ is open and $Z \cap Y$ is closed in $X$, then $Z \subseteq Y$.

Proof Let $\tau_{Z}$ denote the subspace topology on $Z$ and let $\mathbf{C}_{Z}$ denote the closure operator in $\left\langle Z, \tau_{Z}\right\rangle$. Since $Z$ is open in $\langle X, \tau\rangle$, we have $Z \cap Y$ is dense in $\left\langle Z, \tau_{Z}\right\rangle$. Therefore, $\mathbf{C}_{Z}(Z \cap Y)=Z$. Because $Z \cap Y$ is closed in $\langle X, \tau\rangle$, it is also closed in $\left\langle Z, \tau_{Z}\right\rangle$. Thus, $Z \cap Y=\mathbf{C}_{Z}(Z \cap Y)=Z$. It follows that $Z \subseteq Y$.

Lemma 4.10 Let $A$ be a Heyting algebra and let $X$ be its Esakia space. If $\bar{A} \simeq \widehat{A}$, then $X_{0}=X_{\text {iso }} \subseteq X_{\text {fin. }}$. Consequently, $X_{\text {fin }}$ is dense and $A$ is finitely approximable.

Proof Since $\bar{A} \simeq \widehat{A}$, it follows from Theorem 4.4.1 that $X_{0}$ is dense in $X$, and that $\left\langle X_{0}, \leq\right\rangle$ is order-isomorphic to $\left\langle X_{\mathrm{fin}}, \leq\right\rangle$. As $X_{0}$ is dense in $X$, by Lemma 3.6, $X_{0}=X_{\text {iso. }}$. From $\left\langle X_{0}, \leq\right\rangle \simeq\left\langle X_{\text {fin }}, \leq\right\rangle$ it follows that $\uparrow x \cap X_{0}$ is finite for each $x \in X_{0}$. Since $X$ is Hausdorff, $\uparrow x \cap X_{0}$ is closed. Moreover, because $x \in X_{0}, \uparrow x$ is open by definition; so by Lemma 4.9, $\uparrow x \subseteq X_{0}$. Therefore, $\uparrow x=\uparrow x \cap X_{0}$ is finite, whence $x \in X_{\text {fin }}$. It follows that $X_{0} \subseteq X_{\text {fin }}$, so $X_{\text {fin }}$ is dense, and so, by [2, Theorem 3.1], $A$ is finitely approximable. 
Note that $\bar{A} \simeq \widehat{A}$ does not imply that $X_{0}$ is equal to $X_{\text {fin }}$ as the following example shows.

Example 4.11 Let $B$ be the Boolean algebra of finite and cofinite subsets of $\omega$ and let $X$ be its Stone space. Then $X$ is homeomorphic to the one-point compactification of $\omega$ (see Fig. 3), $X_{0}=X_{\text {iso }}=\omega$ and it is a proper subset of $X_{\text {fin }}=X$. On the other hand, both $\bar{B}$ and $\widehat{B}$ are isomorphic to the powerset of $\omega$. Since $B^{\sigma} \simeq \mathcal{P}(X) \simeq \mathcal{P}(\omega)$, because both $\omega$ and $X$ are countable, it follows that $\bar{B} \simeq \widehat{B} \simeq B^{\sigma}$.

Lemma 4.12 Let $A$ be a Heyting algebra and let $X$ be its Esakia space. Then the following conditions are equivalent:

(1) $X_{\text {fin }} \subseteq X_{0}$.

(2) Each filter of $A$ of finite index is principal.

Proof (1) $\Rightarrow(2)$ : Let $F$ be a filter of $A$ and let $C_{F}$ be the corresponding closed upset of $X$. If $F$ is of finite index, then $A / \theta_{F}$ is finite, and so $C_{F}$ is also finite. Therefore, by (1), $C_{F}$ is a finite subset of $X_{0}$, so is clopen. Thus, $F$ is a principal filter.

$(2) \Rightarrow(1)$ : Let $x \in X_{\text {fin }}$. Then $\uparrow x$ is a finite closed upset of $X$. Therefore, the corresponding filter of $A$ is of finite index, hence principal by (2). It follows that $\uparrow x$ is a finite clopen upset of $X$. Thus, $x$ is an isolated point of $X$ and $\uparrow x$ is clopen, so $x \in X_{0}$.

As follows from [4, Chapter 3], if $A$ is the free $n$-generated Heyting algebra and $X$ is its Esakia space, then $X_{\text {fin }} \subseteq X_{0}$, so each filter of $A$ of finite index is principal.

Theorem 4.13 Let $A$ be a Heyting algebra and let $X$ be its Esakia space. Then the following conditions are equivalent:

(1) There is an isomorphism of completions $\gamma:(\bar{A}, \eta) \rightarrow(\widehat{A}, \iota)$.

(2) $X_{0}$ is dense and $X_{0}=X_{\text {fin. }}$.

(3) A is finitely approximable and each filter of $A$ of finite index is principal.

Proof $(1) \Rightarrow(2):$ Suppose that there is an isomorphism $\gamma: \bar{A} \rightarrow \widehat{A}$ such that $\gamma \circ \eta=$ ı. Then $\bar{A} \simeq \widehat{A}$, so by Theorem 4.4 and Lemma 4.10, $X_{0}$ is dense and $X_{0} \subseteq X_{\text {fin }}$. It is left to be shown that $X_{\text {fin }} \subseteq X_{0}$. By Lemma 4.2, $(\bar{A}, \eta) \simeq\left(\mathrm{Up}\left(X_{0}\right), \varphi_{0}\right)$ and $(\widehat{A}, \iota) \simeq\left(\mathrm{Up}\left(X_{\mathrm{fin}}\right), \varphi_{\mathrm{fin}}\right)$. Therefore, $\gamma \circ \eta=\iota$ implies there is a lattice isomorphism $\lambda: \operatorname{Up}\left(X_{0}\right) \rightarrow \operatorname{Up}\left(X_{\text {fin }}\right)$ such that $\lambda \circ \varphi_{0}=\varphi_{\text {fin }}$. Let $x \in X_{\text {fin }}$. Then $\uparrow x \in \operatorname{Up}\left(X_{\text {fin }}\right)$. Since $\lambda$ is onto, there is $U \in \operatorname{Up}\left(X_{0}\right)$ such that $\lambda(U)=\uparrow x$. We have $U=\bigcup\left\{\uparrow y \cap X_{0} \mid\right.$ $y \in U$ \}. From $y \in X_{0}$ it follows that $\uparrow y$ is a clopen upset of $X$. Therefore, there is $a_{y} \in A$ such that $\uparrow y=\varphi\left(a_{y}\right)$. Thus,

$$
\begin{aligned}
U & =\bigcup\left\{\uparrow y \cap X_{0} \mid y \in U\right\} \\
& =\bigcup\left\{\varphi\left(a_{y}\right) \cap X_{0} \mid y \in U\right\} \\
& =\bigcup\left\{\varphi_{0}\left(a_{y}\right) \mid y \in U\right\},
\end{aligned}
$$


and so

$$
\begin{aligned}
\uparrow x & =\lambda(U) \\
& =\lambda\left(\bigcup\left\{\varphi_{0}\left(a_{y}\right) \mid y \in U\right\}\right) \\
& \left.=\bigcup\left\{\lambda \varphi_{0}\left(a_{y}\right) \mid y \in U\right\}\right) \\
& \left.=\bigcup\left\{\varphi_{\text {fin }}\left(a_{y}\right) \mid y \in U\right\}\right) .
\end{aligned}
$$

Consequently, there is $y \in X_{0}$ such that $\uparrow x=\varphi_{\text {fin }}\left(a_{y}\right)=\varphi\left(a_{y}\right) \cap X_{\text {fin }}=\uparrow y \cap$ $X_{\text {fin }}=\uparrow y$ since $y \in X_{0} \subseteq X_{\text {fin }}$. It follows that $x=y$, so $x \in X_{0}$, and so $X_{0}=X_{\text {fin }}$.

$(2) \Rightarrow(1)$ : If $X_{0}$ is dense, then by Theorem 3.7, there is an isomorphism $f: \bar{A} \rightarrow$ $\operatorname{Up}\left(X_{0}\right)$ such that $f \circ \eta=\varphi_{0}$. From $X_{0}=X_{\text {fin }}$ it follows that $X_{\text {fin }}$ is dense, $\operatorname{Up}\left(X_{0}\right)=$ $\mathrm{Up}\left(X_{\text {fin }}\right)$, and $\varphi_{0}=\varphi_{\text {fin }}$. By Lemma 4.2 , there is an isomorphism $h: \widehat{A} \rightarrow \mathrm{Up}\left(X_{\text {fin }}\right)$ such that $h \circ \iota=\varphi_{\text {fin. }}$. Let $\gamma=h^{-1} \circ f$. Then $\gamma: \bar{A} \rightarrow \widehat{A}$ is an isomorphism such that $\gamma \circ \eta=\imath$.

$(2) \Rightarrow(3)$ : If $X_{0}$ is dense and $X_{0}=X_{\text {fin }}$, then $X_{\text {fin }}$ is also dense, hence $A$ is finitely approximable by [2, Theorem 3.1]. Moreover, $X_{\text {fin }} \subseteq X_{0}$ implies, by Lemma 4.12, that each filter of $A$ of finite index is principal.

(3) $\Rightarrow(2)$ : If $A$ is finitely approximable, then $X_{\text {fin }}$ is dense by [2, Theorem 3.1]. Therefore, $X_{0} \subseteq X_{\text {iso }} \subseteq X_{\text {fin }}$. Moreover, since each filter of $A$ of finite index is principal, by Lemma $4.12, X_{\text {fin }} \subseteq X_{0}$. Thus, $X_{0}=X_{\text {fin }}$ and $X_{0}$ is dense.

Among the examples of Heyting algebras that satisfy conditions of Theorem 4.13 are finitely generated finitely approximable Heyting algebras (cf. Corollary 4.6.3). Thus, for finitely generated finitely approximable Heyting algebras $A$, the strengthening of Corollary 4.6.3 to the isomorphism of $\bar{A}$ and $\widehat{A}$ that commutes with $\eta$ and $\iota$ is an immediate consequence of Theorem 4.13. Another immediate consequence of Theorems 4.13 and 4.8 is the following:

Corollary 4.14 For a Heyting algebra $A,(\bar{A}, \eta) \simeq\left(A^{\sigma}, \zeta\right) \simeq(\widehat{A}, \iota)$ iff $A$ is finite. In particular, if $A$ is a Boolean algebra, then $(\bar{A}, \eta) \simeq\left(A^{\sigma}, \zeta\right) \simeq(\widehat{A}, \iota)$ iff $A$ is finite.

We conclude by summarizing the main theorems of this section.

Corollary 4.15 Let $A$ be a Heyting algebra.

(1) (a) $\bar{A} \simeq A^{\sigma}$ iff $A$ is completely join-prime generated and $\left\langle J^{\infty}(A), \geq\right\rangle$ is orderisomorphic to the poset of prime filters of $A$.

(b) $(\bar{A}, \eta) \simeq\left(A^{\sigma}, \zeta\right)$ iff $A$ is finite.

(2) (a) $\bar{A} \simeq \widehat{A}$ iff $A$ is completely join-prime generated and $\left\langle J^{\infty}(A), \geq\right\rangle$ is orderisomorphic to the poset of prime filters of $A$ of finite index.

(b) $(\bar{A}, \eta) \simeq(\widehat{A}, \iota)$ iff $A$ is finitely approximable and each filter of $A$ of finite index is principal.

(3) $A^{\sigma} \simeq \widehat{A}$ iff $\left(A^{\sigma}, \zeta\right) \simeq(\widehat{A}$, ı) iff each prime filter of $A$ is of finite index.

(4) (a) $\bar{A} \simeq A^{\sigma} \simeq \widehat{A}$ iff $A$ is completely join-prime generated, $\left\langle J^{\infty}(A), \geq\right\rangle$ is isomorphic to the poset of prime filters of $A$, and each prime filter of $A$ is of finite index.

(b) $(\bar{A}, \eta) \simeq\left(A^{\sigma}, \zeta\right) \simeq(\widehat{A}, \iota)$ iff $A$ is finite. 


\section{Further Directions}

The results of Section 4 can easily be modified to apply to modal algebras in light of the fact that the Jónsson-Tarski duality for modal algebras is essentially the Esakia duality formulated for modal algebras. The modal algebra case of Theorem 4.13 and Corollary 4.6.3 will be described in detail in [20].

More generally, using the duality theory developed by Goldblatt [11], we would expect that our main results generalize to the case of distributive lattices with operators. In fact, such results as those in [8] and [12] indicate that distributivity of the underlying lattice may not be essential at all.

Acknowledgements We are greatly indebted to the referee for many valuable suggestions. More specifically, the notion of a principally separated lattice (Definition 2.4.1) and the fact that there exist (incomplete) completely join-prime generated lattices, which are not principally separated (Example 2.3) were suggested by the referee. In addition, his/her comments resulted in the reformulations of Lemmas 3.2 and 4.2 and Theorems 3.4, 3.5, and 3.7. He/she also pointed out some inaccuracies in the original proofs of Theorems 3.7, 3.14, and 4.13 .

Open Access This article is distributed under the terms of the Creative Commons Attribution Noncommercial License which permits any noncommercial use, distribution, and reproduction in any medium, provided the original author(s) and source are credited.

\section{References}

1. Banaschewski, B., Bruns, G.: Categorical characterization of the MacNeille completion. Arch. Math. 18, 369-377 (1967)

2. Bezhanishvili, G., Bezhanishvili, N.: Profinite Heyting algebras. Order 25, 211-227 (2008). http://www.cs.le.ac.uk/people/nb118/Publications/Bezh-Bezh-final.pdf

3. Bezhanishvili, G., Gehrke, M., Mines, R., Morandi, P.J.: Profinite completions and canonical extensions of Heyting algebras. Order 23, 143-161 (2006)

4. Bezhanishvili, N.: Lattices of intermediate and cylindric modal logics. PhD thesis, University of Amsterdam (2006)

5. Erné, M.: Bigeneration in complete lattices and principal separation in ordered sets. Order 8(2), 197-221 (1991)

6. Erné, M.: The Dedekind-MacNeille completion as a reflector. Order 8(2), 159-173 (1991)

7. Esakia, L.L.: Topological Kripke models. Sov. Math., Dokl. 15, 147-151 (1974)

8. Gehrke, M., Harding, J.: Bounded lattice expansions. J. Algebra 239, 345-371 (2001)

9. Gehrke, M., Harding, J., Venema, Y.: MacNeille completions and canonical extensions. Trans. Am. Math. Soc. 358, 573-590 (2006)

10. Gehrke, M., Jónsson, B.: Bounded distributive lattices with operators. Math. Jpn. 2, 207-215 (1994)

11. Goldblatt, R.: Varieties of complex algebras. Ann. Pure Appl. Logic 44, 173-242 (1989)

12. Harding, J.: On profinite completions and canonical extensions. Algebra Univers. 55, 293-296 (2006)

13. Harding, J., Bezhanishvili, G.: MacNeille completions of Heyting algebras. Houst. J. Math. 30, 937-952 (2004)

14. Jónsson, B., Tarski, A.: Boolean algebras with operators. Part I. Am. J. Math. 73, 891-939 (1951)

15. McKenzie, R.: Equational bases and nonmodular lattice varieties. Trans. Am. Math. Soc. 174, $1-43(1972)$

16. Ono, H.: Algebraic semantics for predicate logics and their completeness. In: Logic at work. Stud. Fuzziness Soft Comput., vol. 24, pp. 637-650. Physica, Heidelberg (1999)

17. Ono, H.: Completions of algebras and completeness of modal and substructural logics. In: Advances in modal logic, vol. 4, pp. 335-353. King's Coll. Publ., London (2003) 
18. Priestley, H.A.: Representation of distributive lattices by means of ordered Stone spaces. Bull. Lond. Math. Soc. 2, 186-190 (1970)

19. Rasiowa, H., Sikorski, R.: The Mathematics of Metamathematics. Monografie Matematyczne, Tom 41. Państwowe Wydawnictwo Naukowe, Warsaw (1963)

20. Vosmaer, J.: MacNeille completion and profinite completion can coincide on finitely generated modal algebras. Algebra Univers. (2008, in press)

21. Whitman, P.M.: Splittings of a lattice. Am. J. Math. 65, 179-196 (1943) 